\title{
A REPORT OF TWO CASES OF SARCOIDOSIS WITH BRONCHIAL CARCINOMA
}

\author{
BY
}

\author{
MICHAEL JEFFERSON, W. T. SMITH, A. BRIAN TAYLOR, AND K. VALTERIS \\ From the Queen Elizabeth Hospital, Birmingham, and the Department of Pathology, Birmingham University
}

(RECEIVED FOR PUBLICATION JUNE 29, 1954)

The chance discovery of two patients in whom generalized sarcoidosis was associated with carcinoma of the lung appears worth reporting, since the association has seemingly not been recorded before. In each case the patient had been a known sufferer from sarcoidosis for a number of years, and in neither was the later development of pulmonary cancer suspected clinically.

\section{CAse Histories}

CASE 1.-John C., aged 52, an industrial engineer, was admitted on November 19, 1951, to the Queen Elizabeth Hospital, Birmingham, under the care of one of us (A. B. T.). His illness had begun in 1943 with the appearance of a small nodular swelling on the right shin. The eruption lasted some months, and a radiograph of the leg was said to have shown a periosteal reaction underlying it. No diagnosis was made, and it finally subsided without treatment. In the course of the next two years, similar indolent swellings came and went on either leg until one or two broke down to form chronic ulcers. He was then treated in hospital with a course of penicillin which resulted in their healing. The blood Wassermann reaction was negative, and it was suggested that the skin lesions were those of Bazin's disease.

Early in 1947 at mass radiography at his works some shadow in the lung had been seen, but since he was free of chest symptoms and in good general health no further action was taken then. Eighteen months later he began to complain of slight pain in the left side of the chest, breathlessness, and productive morning cough. He was first seen, because of these symptoms, as an out-patient in August, 1948. There were scars on both legs from old ulceration, and he stated that occasional lumps had appeared on his legs since he was in hospital in 1945. Clinical examination was otherwise negative. Blood pressure was $120 / 70 \mathrm{~mm}$. Hg. Radiography of the chest disclosed enlarged hilar shadows bilaterally and increased lung markings in both lower zones (Fig. 1), and a tentative diagnosis of pulmonary sarcoidosis was made. A year later, his only complaint was morning cough with sputum, and a radiograph showed no significant change from the previous film. The Mantoux reaction was negative at 1 in 1,000. A dermatological opinion about the leg eruptions was sought, and their sarcoid nature affirmed: after this a confident diagnosis of systemic sarcoidosis with pulmonary and cutaneous manifestations was made.

At a further out-patient attendance in February, 1950 , productive cough was still his only complaint, though swellings on the legs were still recurring from time to time. The chest film again showed no definite change. A year later (January, 1951) he was becoming more easily breathless and coughing more than before. There were now rales at both bases, but radiologically the hilar masses were as before and there was still no distinct change in the lung fields. In May, 1951, his condition was stationary; the Mantoux test was repeated, and was still negative at 1 in 1,000. There was a fresh red indurated patch on the left leg. A radiograph showed a new opacity, suggestive of consolidation in the lingular lobe of the left side (Fig. 2), which was ascribed to progression of the pulmonary sarcoid lesions. By August, 1951, he was finding it hard to keep at work because of mounting shortness of breath, was eating poorly and losing weight, and he mentioned that his sputum was now sometimes slightly blood-stained. His blood pressure had risen to $170 / 100 \mathrm{~mm}$. $\mathrm{Hg}$, and his pulse was rapid. This further deterioration in his condition was again taken to mean increasing sarcoidosis of the lungs, with consequent embarrassment of the pulmonary circulation.

In mid-September, 1951, the patient went to North Wales on holiday, and while away developed a fever $\left(103^{\circ} \mathrm{F}\right.$.) and signs of bronchopneumonia with left-sided pleurisy. He failed to respond satisfactorily to hospital treatment at Rhyl, and was ultimately transferred to the Queen Elizabeth Hospital, as mentioned above, on November 19, 1951. On admission he was gravely ill, dyspnoeic, with distended neck veins and oedematous ankles, and clinical signs of an extensive left pleural effusion; the radiograph of the chest is shown in Fig. 3. Aspiration of the chest, antibiotics, and continuous oxygen inhalation by mask failed to give him relief. He lapsed into coma on the afternoon of November 29, and died 12 hours later.

Necropsy.-Necropsy was performed on November 30. The body weighed $62 \mathrm{~kg}$. The legs were oedematous, with patchy brownish pigmentation and induration of the skin on the anterior surfaces below the knees.

The mouth, oesophagus, larynx, and trachea were normal. 


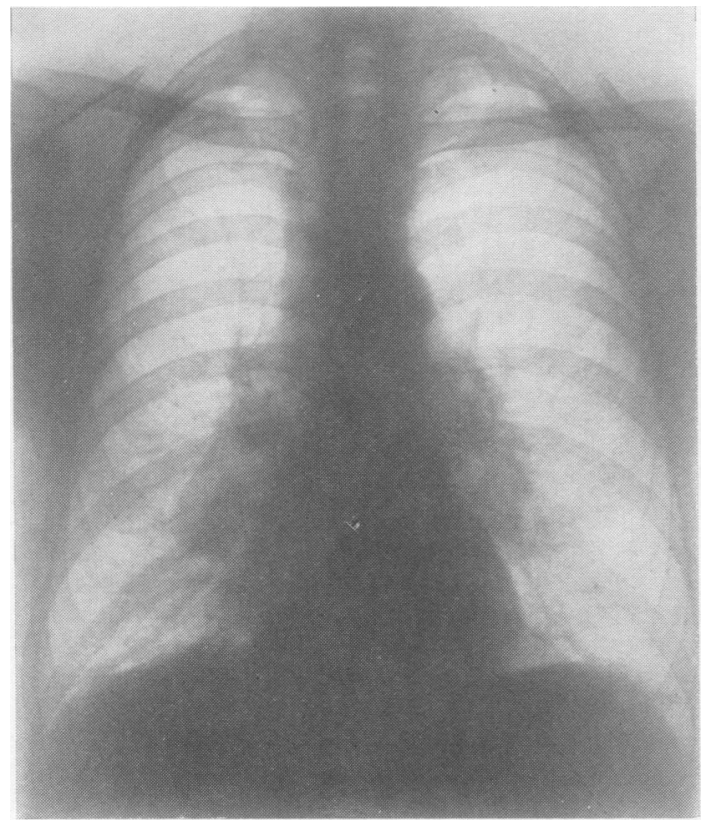

Fig. 1.-Chest radiograph in August, 1948, showing bilaterally enlarged hilar masses.

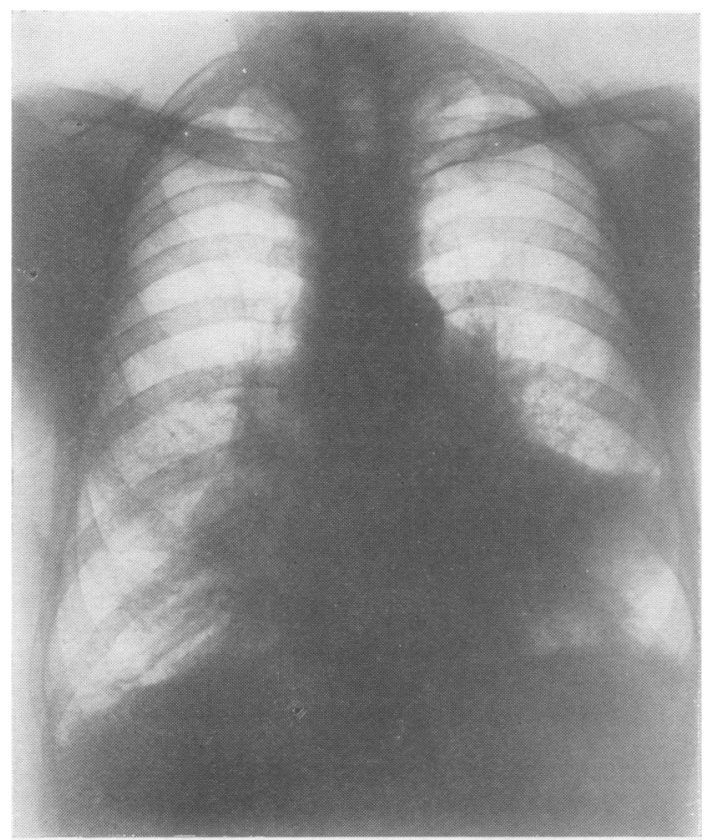

FIG. 2.-Chest radiograph in May, 1951, now showing opacity in the left lung field.

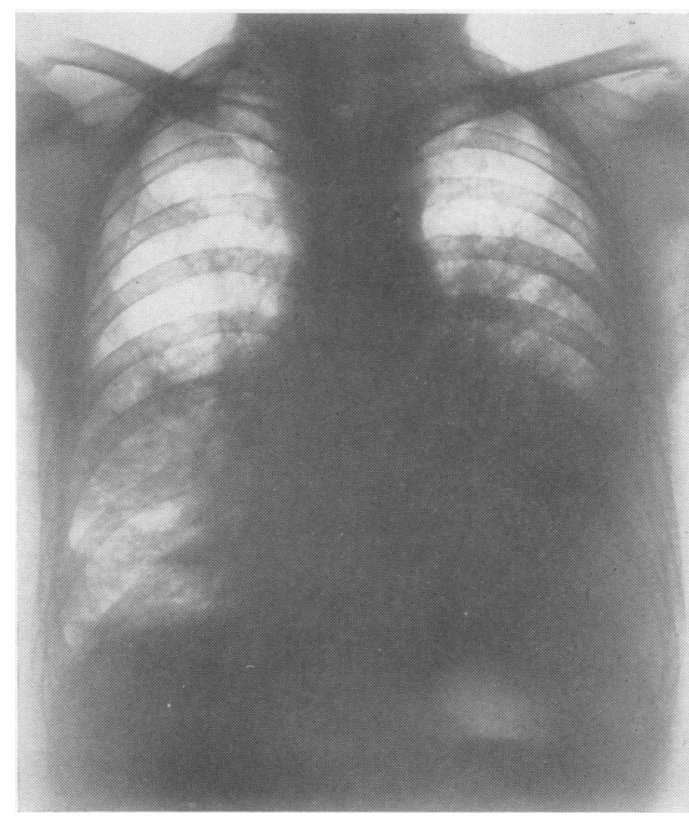

FIG. 3 (Case 1).-Chest radiograph in November, 1951, terminal state.

In the lungs there were dense bilateral pleural adhesions, with fibrinous and fibrino-haemorrhagic exudate $\varrho$ between adherent surfaces; the left parietal pleura was $\overrightarrow{\vec{O}}$ markedly thickened. Both visceral pleurae were studded 3 with many firm, grey, coarsely granular plaques, up to $\supsetneq$ $1 \mathrm{~cm}$. in diameter, more numerous on the left side, and $\bar{c}$ confluent in places. Firm masses of similar tissue, but more yellow, infiltrated both pleural apices. The main left lower lobe bronchus was almost totally obstructed by firm, grey-white neoplasm infiltrating through the bronchial wall. A frozen section showed this to be keratinizing squamous carcinoma. Below the obstruction the bronchus was dilated and filled with mucopus, and the left lower lobe showed suppurative pneumonia. Both lungs were unusually firm on palpation, and cut 을 surfaces disclosed numerous grey, somewhat striated, $\supset$ areas of dense fibrosis. and a few smaller more discrete white nodules of neoplastic tissue. Much diffuse anthracotic pigmentation was visible. Hilar and peri- or bronchial lymph nodes were markedly enlarged; the $N$ cut surfaces were predominantly grey with some smaller N paler foci of secondary carcinoma.

The heart and main blood vessels were normal.

In the stomach there was an ulcerated tumour deposit, 0 $4 \times 0.5 \mathrm{~cm}$., in the mucosa of the anterior wall of the $\Phi$ fundus. The perigastric lymph nodes were enlarged $\stackrel{\mathcal{P}}{+}$ and contained tumour tissue on section.

The liver $\left(1,650 \mathrm{~g}\right.$.) was softer and paler than normal $\bar{o}^{\circ}$ and the lobular pattern was blurred on the cut surface. $\stackrel{\mathbb{D}}{ }$ The bile ducts were patent and the gall bladder normal. $\frac{?}{\mathbb{8}}$

The spleen $(300 \mathrm{~g}$.) had abundant miliary nodules on $\varrho$ the cut surface, and also a larger grey-white nodule of secondary carcinoma (Fig. 4). 


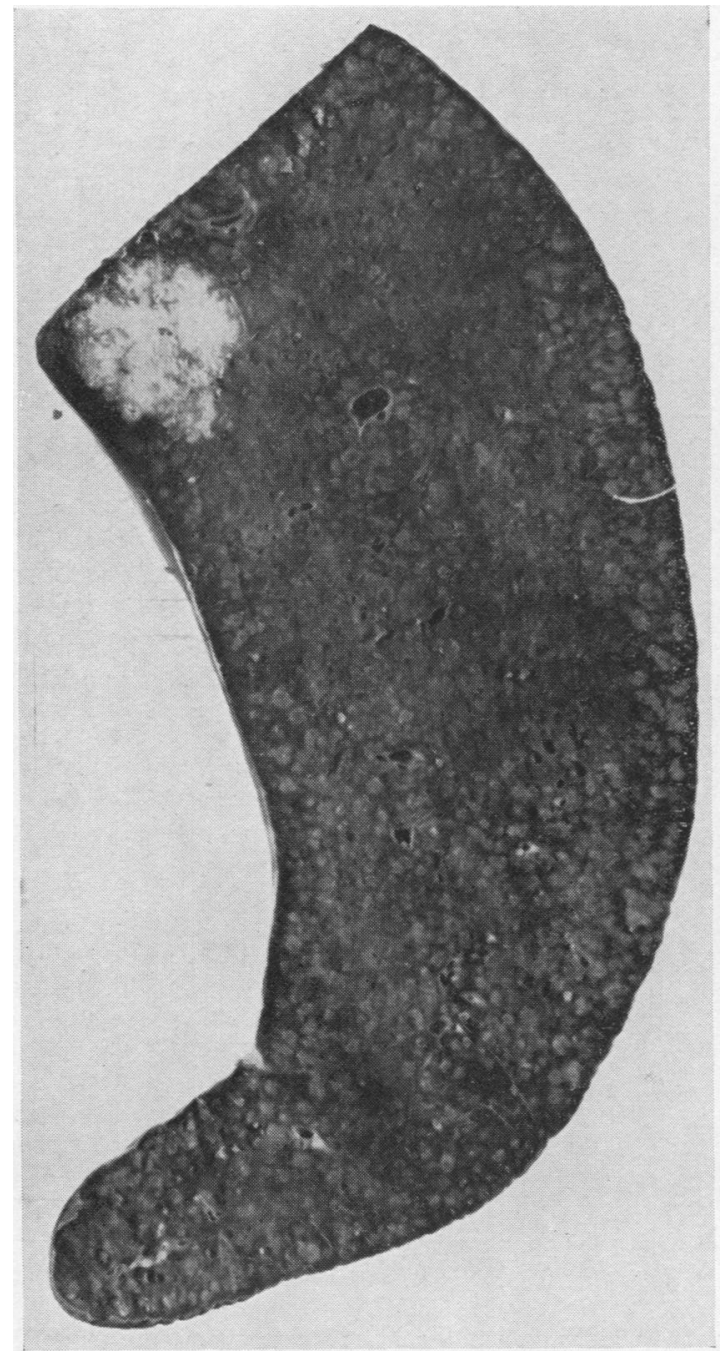

FIG. 4.-Spleen showing fine stippling of cut surface with sarcoid nodules. A single larger deposit of secondary carcinoma, approximately $1 \mathrm{~cm}$. in diameter, is seen at one pole.

The oesophagus, intestines, and pancreas were normal. The kidneys (400 g.) were congested.

The bladder, prostate, restes, suprarenals, thyroid, pituitary, brain, and left femur were normal. The pineal gland was larger than normal.

Histology.-There was a keratinizing squamous carcinoma of the left lower lobe bronchus, infiltrating the surrounding lung by direct spread and permeating the peribronchial and subpleural lymphatics and also small blood vessels. Many carcinomatous foci show central necrosis. In addition there are non-neoplastic areas showing non-caseating epithelioid follicular lesions with the histological characters of sarcoidosis; multinucleated giant cells are numerous. These sarcoid lesions show varying degrees of hyaline fibrosis (Fig. 5). Acid-fast bacilli are not present. Diffuse interstitial fibrosis, congestion, and focal areas of bronchopneumonia and collapse are also seen. The pleural plaques are carcinomatous deposits.

In the hilar lymph nodes sarcoid lesions are extremely numerous; many show dense hyaline fibrosis. Small foci of secondary squamous carcinoma are also seen (Fig. 6).

In the spleen are numerous sarcoid follicles, mostly arranged in groups, both in the pulp and in the Malpighian bodies. Some show complete hyaline fibrosis; in others an earlier stage of the process can be seen with fibrosis starting at the periphery of the lesions (Fig. 7).

In the skin (taken from the site of the previous leg ulceration) there is an oleo-granulomatous reaction in the deeper dermis, which is not typical of sarcoidosis, but resembles more a "foreign-body" reaction, perhaps resulting from previous local medication.

In the stomach the carcinomatous metastasis is confirmed.

No significant histological changes are found in the tonsils, thyroid, pituitary, suprarenal, pineal, submandibular salivary gland, and myocardium.

Bacteriological examinations for tubercle bacilli by culture and animal inoculation were negative.

In this case the symptoms and course of the earlier phases of the patient's illness can leave little room for doubt that the clinical diagnosis of sarcoidosis was correct, and indeed it was fully confirmed by the post-mortem findings. When the patient began to complain, about a year before his death, of increasing respiratory symptoms and the chest radiograph eventually revealed new features, the deterioration was attributed to progression of the sarcoid disease and the possibility of developing pulmonary neoplasm was not considered. In retrospect it is improbable that the growth could have been removed before secondary dissemination occurred, even had suspicion of cancer been aroused by the chest film taken in May.

CASE 2.-Thomas B., aged 45, a commercial traveller, was admitted on April 18, 1953, to the Neurological Unit of the Queen Elizabeth Hospital. The story of his illness extended over a good many years, his first symptoms-undue shortness of breath on exertion and general debility - having developed in 1939 and gradually receded again without his seeking medical attention. In January, 1946, he had an illness diagnosed as acute bronchitis, which kept him in bed for six weeks. About a year later (1947) his chest was screened in a mass radiography survey of his home town, and the diagnosis of sarcoidosis was first suggested then. In March, 1948, he developed a swelling of the right ear, biopsy of which showed typical sarcoid lesions. During the next three years he slowly became more disabled by ill-health, and he was admitted to hospital for investigation in May, 1951. At this time there was radiological evidence of pulmonary sarcoidosis and a chronic per- 
nioid condition of the right ear; the Mantoux test was negative, and radiographs of the hands were normal. Treatment with breathing exercises was given, but after leaving hospital he still found himself troubled by cough, breathlessness, and fatigue.

In November, 1952, he was again admitted to hospital. A chest radiograph showed advanced pulmonary fibrosis and secondary emphysema (Fig. 8), but radiographs of the hands were still normal. The Mantoux test was negative up to 1 in 100. No tubercle bacilli were found on repeated sputum examinations. Total plasma protein was $7.1 \mathrm{~g}$. (albumin 2.8 g., globulin 4.3 g.). Cortisone, $50 \mathrm{mg}$. daily, was given for four weeks, followed by $25 \mathrm{mg}$. daily for a further two weeks. This led to the usual sense of well-being, but radiographs taken at the end of treatment disclosed no radiological improvement in the lungs. The patient was discharged from hospital at the end of December, 1952.

A month later, while at work he lost consciousness without warning, and a few days later, early in February, 1953, had a second similar attack. He began to complain of forgetfulness and what he called "difficulty in thinking," and noticed slight

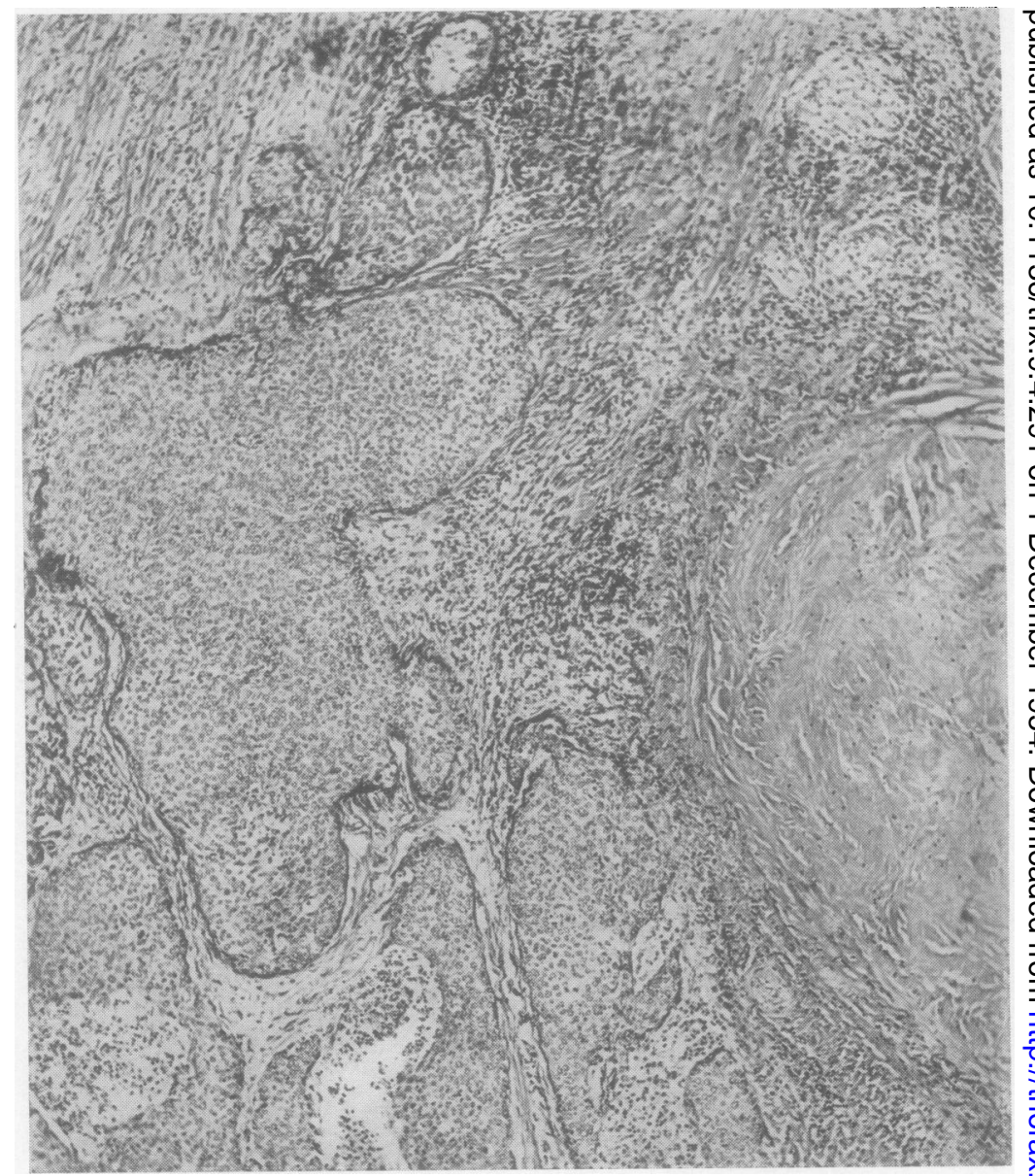

FIG. 5.-Lung of Case 1 showing squamous carcinoma infiltrating the edge of an old hyalinized area of sarcoidosis. Haematoxylin and van Gieson $(\times 50)$ loss of facility in the use of his right arm. Because of these new symptoms he was readmitted to hospital in March. No definitely abnormal neurological signs were found, and the cerebrospinal fluid pressure and contents proved to be completely normal. Skull radiographs were also normal.

The patient was first seen by one of us (M. J.) on April 7, 1953. By this time he presented slight but unmistakable signs of dysphasia, and a mild weakness and clumsiness of the right hand and arm of pyramidal type, but there was no papilloedema. The diagnosis appeared to lie between cerebral sarcoid, or (more likely) an unrelated glioma of the left frontal lobe, and arrangements were made for his transfer to Birmingham for further investigation. By the time he arrived, considerable and rapid worsening of his neurological state had occurred. The right arm was totally paralysed, and the right leg and face showed severe weakness. Signs of right-sided sensory loss of cortical type had also developed, and a right-sided homonymous hemianopia, but there was still no papilloedema. The dysphasia was more pronounced, and it had become hard to keep his attention for long because of frequent transient episodes when he would put his left hand abruptly to his forehead, let his eyelids drop and stop speaking. He would then remain with eyes closed, apparently in a con drowsy and confused state from which it was difficult to rouse him, for as much as two or three minutes. Examination of the chest revealed harsh breath sounds $\omega$ at all areas, and the percussion note was hyper-resonant. F There were palpable lymph glands in both axillae and 0 groins. The right ear was red and somewhat shrivelled $\stackrel{\odot}{\overparen{D}}$ in appearance. Ventriculography was carried out and $\stackrel{\mathcal{Q}}{+}$ disclosed a left fronto-parietal tumour. It had been $T$ intended to obtain a biopsy from this lesion at a separate operation, but further radiographs of the chest showed $\mathbb{D}$ that, in addition to the typical sarcoid changes which $\frac{?}{1}$ had not altered as compared with films taken three or $\varrho$ four months earlier, the posterior part of the fourth rib had disappeared completely (Fig. 9). Cerebral 


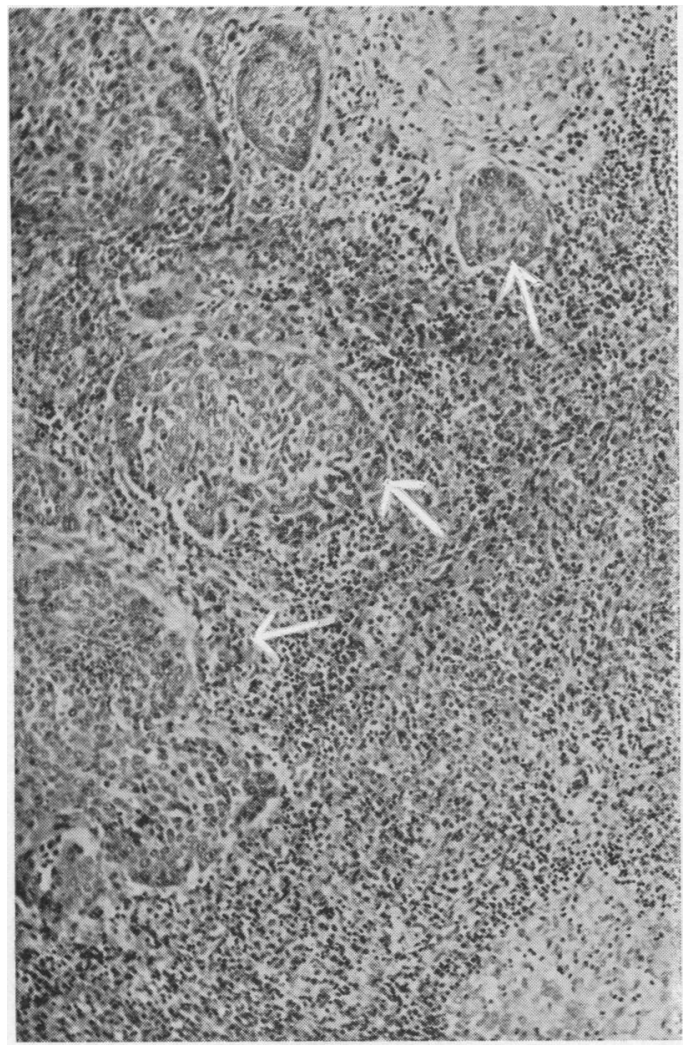

biopsy now did not seem worth while, as it was thought that the intracranial tumour must be metastatic and due to the same primary lesion that had led to the destruction of the rib. Myelomatosis was considered as a possible answer, but a blood film showed no myeloma cells, and sternal puncture gave a normal smear.

Meanwhile the patient was becoming desperately ill. Slight icterus appeared on May 1, deepening in the next few days, and the liver became enlarged two to three finger-breadths below the rib margin; vomiting also developed, and it was believed that these symptoms must be due to hepatic metastases. The level of consciousness steadily declined. On May 8 there was a profuse haematuria, and he died in coma two days later.

Necropsy.-The body weighed $59 \mathrm{~kg}$.; the skin and sclerae were jaundiced; recent biparietal burr-holes were seen. The teeth were carious, and there was suppurative tonsillitis.

The larynx, trachea, and bronchi contained a little mucopus.

There were dense, irregular fibrotic patches on the visceral pleurae, in places puckered and pigmented with carbon. Numerous similar grey and anthracotic zones of fibrosis with clearly defined edges were seen on the cut surfaces of the lungs, mainly in the upper lobes. No caseation or cavitation was seen. Between areas of fibrosis the lung tissue was congested and showed a few tiny creamy-coloured foci of bronchopneumonia. The main right upper lobe bronchus was almost completely occluded by annular carcinoma infiltrating adjacent lung tissue; frozen section showed anaplastic oat-celled carcinoma. Hilar and paratracheal lymph nodes

Fig. 6.-Pulmonary lymph node of Case 1 showing sarcoid follicles. Arrows indicate deposits of secondary carcinoma. Haematoxylin and eosin $(\times 80)$.
Fig. 7.- Spleen of Case 1 showing sarcoid follicles. Note the fibrosis at the periphery of the follicles and the well-marked irregular hyalinization of the stroma of the pulp. Haematoxylin and eosin $(\times 90)$.

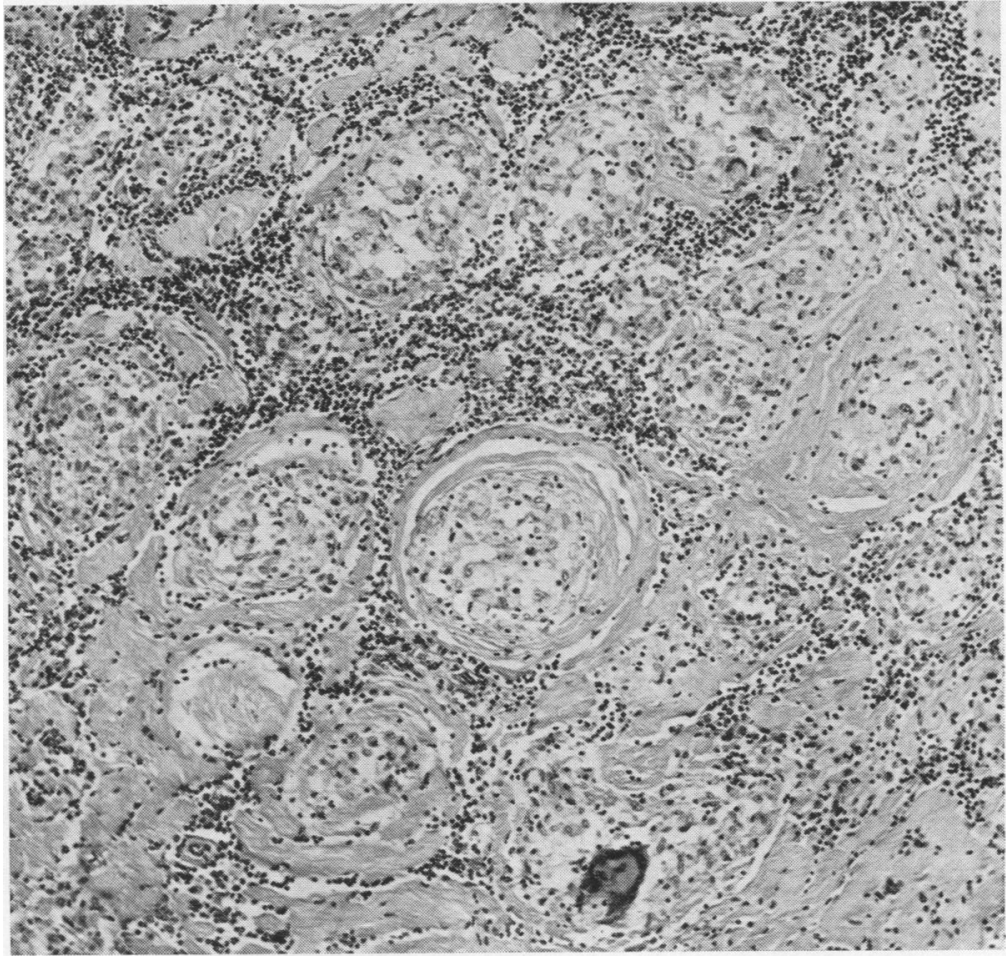


showed marked carbon pigmentation, areas of dense fibrosis and contrasting, softer, paler-coloured zones.

The heart and main thoracic and abdominal blood vessels, the oesophagus, stomach, and intestines were normal.

In the coeliac and mesenteric lymph nodes were areas of fibrosis.

The liver (1,570 g.) was markedly icteric, and showed fatty degeneration. The gall bladder was distended.

The spleen (130 g.) showed a soft inflammatory hyperplasia of the pulp.

The pancreas was much firmer than normal, especially in its head over a smoother area, approximately $1 \mathrm{~cm}$. in diameter. related to the ampulla of Vater. Both the main pancreatic and the common bile duct were obstructed.

Both suprarenals were almost wholly replaced by greyish, partially necrotic, tumour tissue into which haemorrhage had taken place on the left side, resulting in a cyst $8 \mathrm{~cm}$. in diameter filled with firm blood clot.

The upper poles of the kidneys were indented as a result of compression by suprarenal lesions. Cut surfaces showed many whitish nodules $0.5-1.5 \mathrm{~cm}$. in diameter.

The bladder, prostate, testes, thyroid, and pituitary were normal.

The meninges were normal. The convolutional markings of the hemispheres were flattened, especially on the left. The left hemisphere was larger than the right; there was left-sided tentorial herniation, maximum width $1.2 \mathrm{~cm}$., and herniation of the left cingulate gyrus under the free margin of the falx. On coronal sectioning after fixation both hemispheres contained a number of carcinomatous metastases, $0.5-2 \mathrm{~cm}$. in diameter, several showing central necrosis. A large deposit in the left fronto-parietal region measured $8 \mathrm{~cm}$.

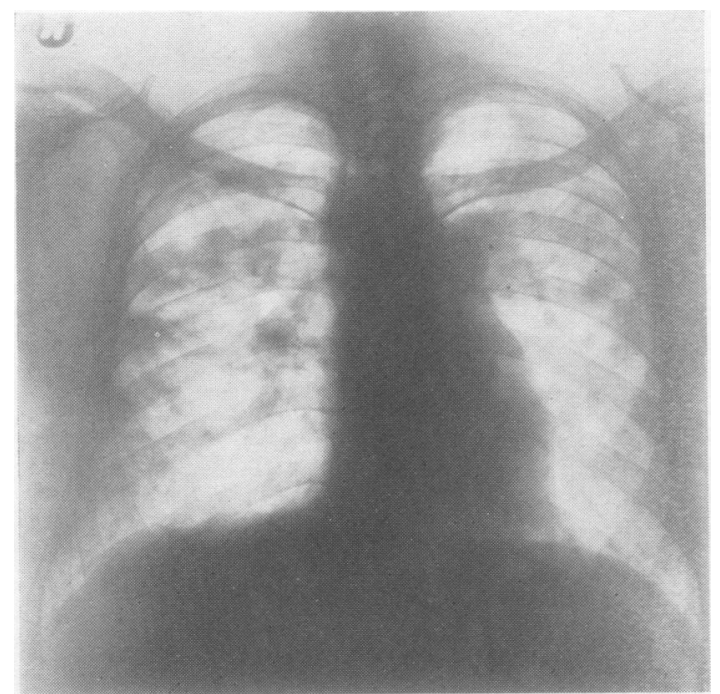

FIG. 8.-Chest radiograph in November, 1952, widespread fibrosis of both lungs.

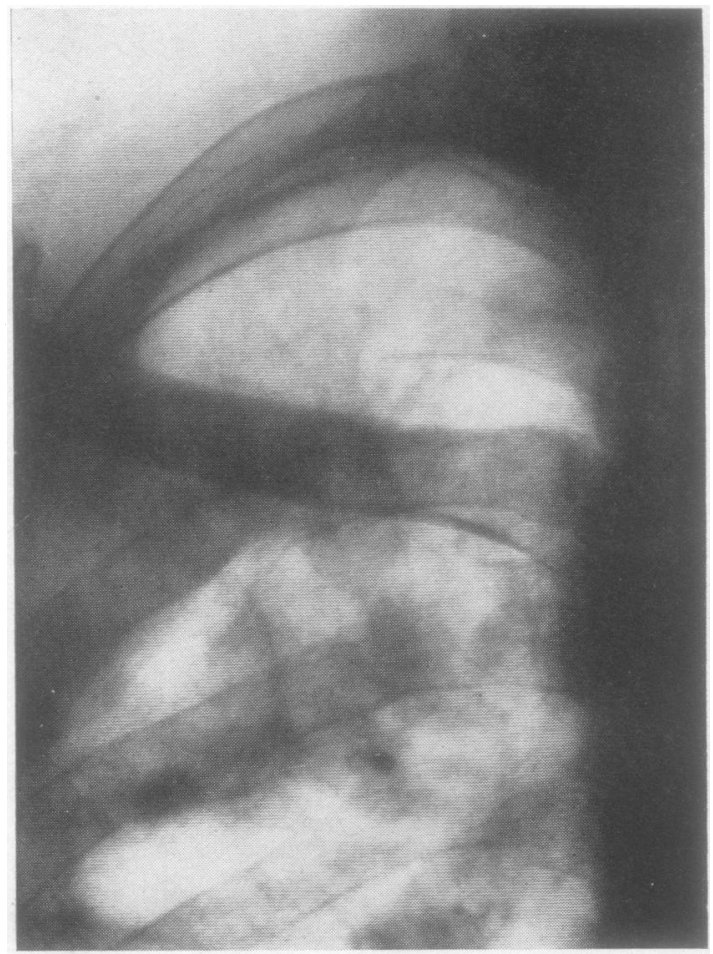

FIG. 9.-Chest in April, 1953, destruction of the right fourth rib posteriorly now evident.

in cross-section. The right cerebellar hemisphere contained a single small deposit. The brain-stem appeared normal. The left femur was normal. The right fourth rib was largely replaced posteriorly by $a \frac{\text { 의 }}{2}$ tumour mass measuring $6 \times 2 \times 1 \mathrm{~cm}$. Animal inocula- $\times$ tion tests and cultures on representative portions of $\frac{0}{3}$ lungs were negative for tubercle bacilli.

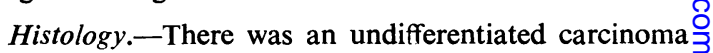
of the right upper lobe bronchus showing much necrosis. There are dense hyaline scars, between which are many을 discrete active sarcoid foci consisting of epithelioid cells $D$ surrounded by lymphocytes and occasional multinucleated giant cells (Fig. 10). The sarcoid follicles N show varying grades of healing by fibrosis and in places ${ }^{-r}$ "fibrinoid" material in their centres. Large irregular N hyaline scars have resulted from coalescence of fibrous N tissue within and between follicles. Some fibrous areas $\omega$ show stippling with haematoxyphile particles. No true caseation or acid-fast bacilli are seen. Some giant cells contain " asteroid bodies" and other inclusions. The $\mathbb{\varnothing}$ rest of the lung shows congestion, emphysema, and zones ${ }^{+}$ of bronchopneumonia.

In the pulmonary lymph nodes are areas of dense $\frac{\overrightarrow{+}}{+}$ fibrous scarring, with occasional giant cells at the peri- $\subseteq$ phery. No secondary carcinoma or active sarcoidosis $\mathbb{Q}$ is seen in sections examined.

The adrenals, kidneys, rib, and brain all show metastatic partially necrotic carcinoma, histologicallyo 


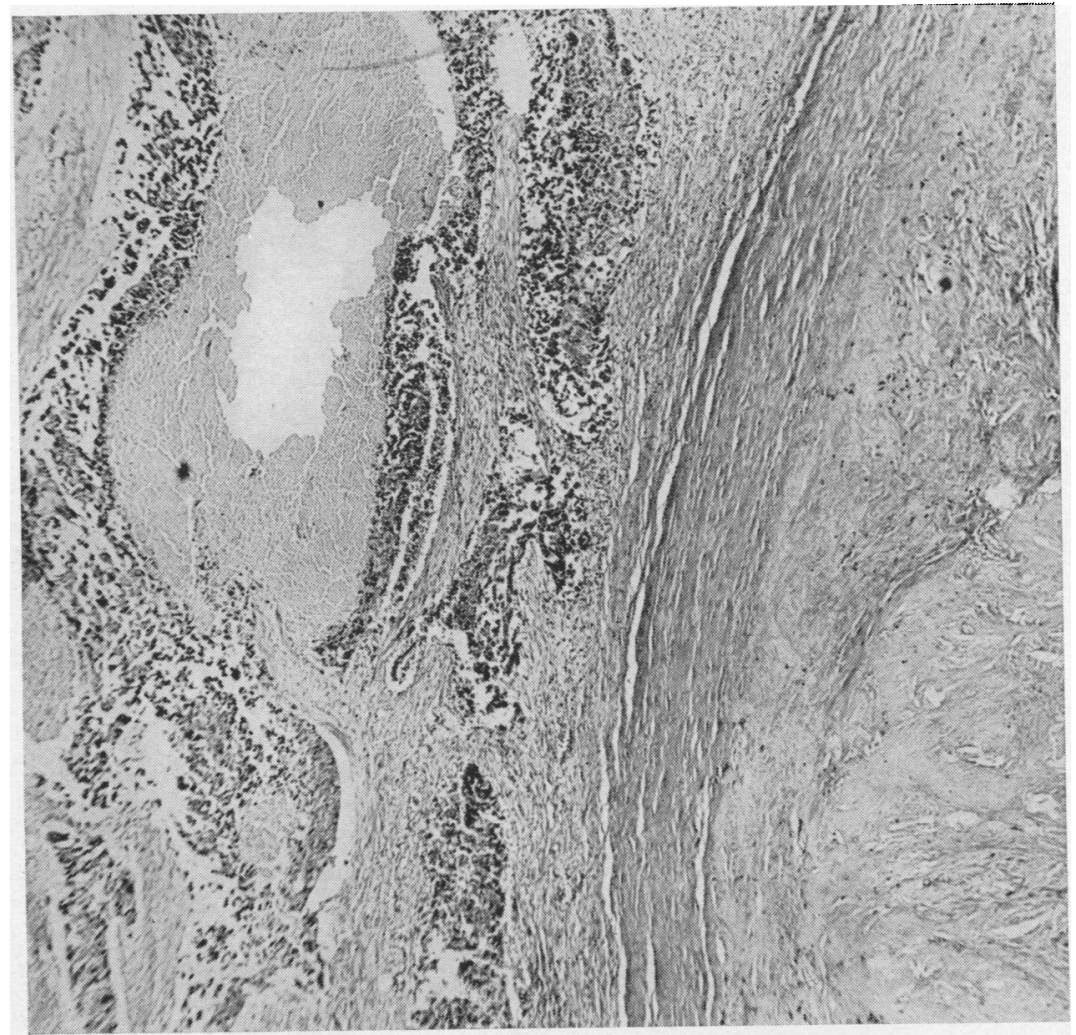

FIG. 10.-Right lung showing anaplastic and partially necrotic bronchial carcinoma adjacent to the edge of a peribronchial lymph node which shows dense collagenous fibrosis. Haematoxylin and van Gieson $(\times 34)$.

Fig. 11.-Liver showing an intralobular sarcoid lesion in which fibrosis is occurring. Epithelioid cells are still recognizable. Haematoxylin and eosin $(\times 168)$.

All photomicrographs are enlarged 1.45 times.

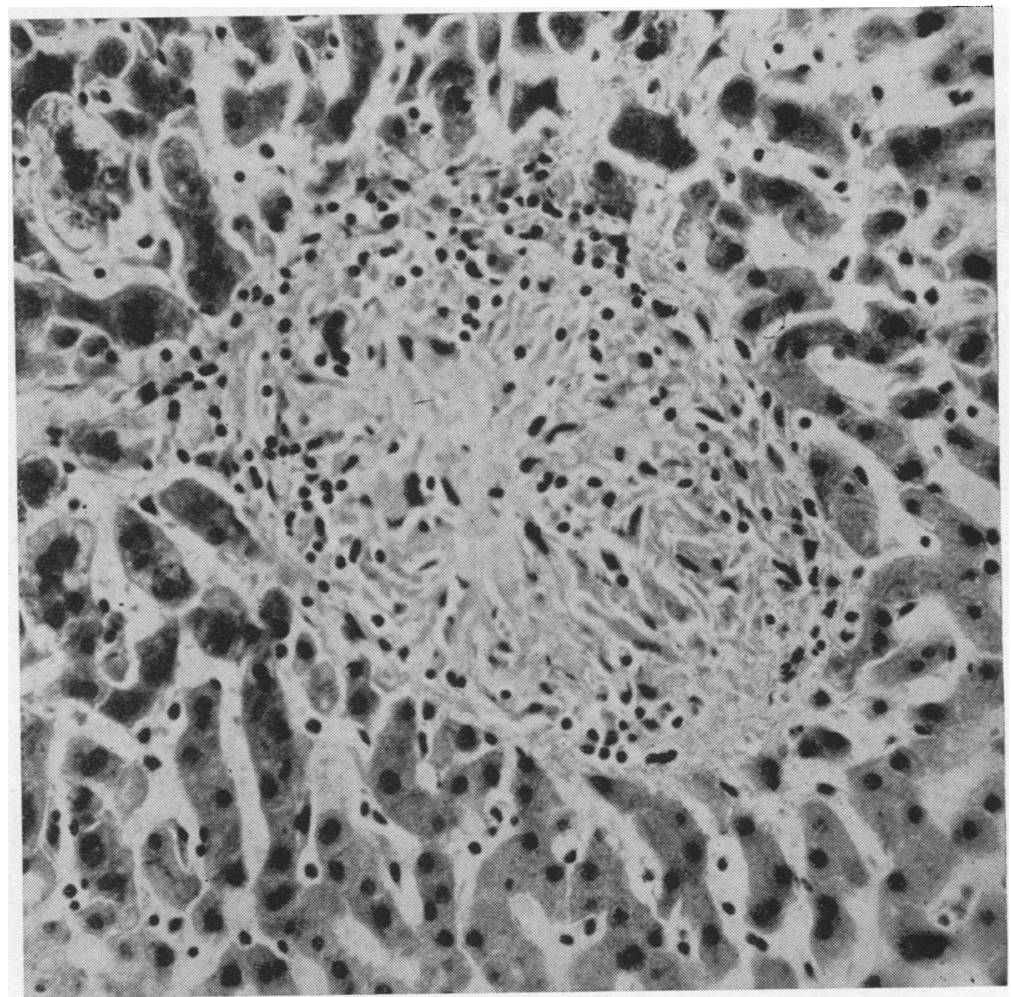


identical with the bronchial growth. The left adrenal also shows areas of dense fibrosis in its substance and in the retroperitoneal connective tissue surrounding it, suggestive of healed sarcoidosis; no active sarcoid lesions are present.

In the coeliac and mesenteric lymph nodes are active and healed sarcoid lesions.

In the liver are seen fatty degeneration, occasional centrilobular necrosis, biliary pigmentation of liver cells, dilatation of bile capillaries, and some portal tract fibrosis. Occasional fibrotic foci associated with scanty epithelioid and giant cells are compatible with sarcoid lesions (Fig. 11).

In the pancreas is well-marked interstitial fibrosis with atrophy of acinar tissue; the islets of Langerhans are relatively preserved. No active sarcoidosis or metastases are seen. The pancreatic atrophy and fibrosis appear to have resulted from duct obstruction by sarcoidosis now healed.

In the cerebral hemispheres and the cerebellum secondary deposits of anaplastic carcinoma resembling the primary are confirmed, but no sarcoid lesions are seen either in the brain or spinal cord. Fibrosis is seen around certain posterior nerve roots with foci suggestive of healing sarcoidosis. The peripheral nerves are normal.

The muscles, pituitary, spleen, and tongue showed no significant changes in the sections examined.

The presence of pulmonary and cutaneous sarcoidosis (the latter histologically proved by biopsy) of many years' duration is established in this case both by the history and by the evidence obtained at necropsy.

Although it was known before the patient died that he was suffering from the effects of metastases, the site of the primary growth was unknown, since it remained radiologically invisible. It is interesting that the jaundice which appeared nine days before death, and was attributed clinically to hepatic secondaries, was in fact obstructive in type, and due to fibrosis in the head of the pancreas.

\section{Discussion}

It is well established that sarcoid-like granulomatous lesions may be found in a wide range of conditions of diverse aetiology. They have been reported in leprosy, tubercle, lues, brucella infections, leishmaniasis, histoplasmosis, Hodgkin's disease, regional ileitis, and Whipple's disease, as well as after exposure to chemical agents, including beryllium, medicat: $d$ oils, and other drugs (Nickerson, 1937; Freiman, 1948; Nadel and Ackerman, 1950). More relevantly, they have also been described in association with neoplasms of several kinds (Nickerson, 1937 ; Anderson, 1942-3 ; Gherardi, 1950; Nadel and Ackerman, 1950; Symmers, 1951; Prior, 1952), either appearing amongst tumour cells at the actual site of the primary growth or more commonly in regional lymph nodes draining the area bearing the neoplasm.

Freiman (1948) has indicated the need for distinguishing between the "sarcoid lesion" and "sarcoidosis," the former implying a tuberculoid granulomatous reaction to the considerable variety of noxious agents just listed as well as to the unknown cause of sarcoidosis itself, and the latter a systematized disease entity with certain welldefined characteristics. The same point is discussed by Nadel and Ackerman (1950), Symmers (1951), and Cowdell (1954). Symmers suggested that, to avoid confusion, the expression sarcoid should be reserved strictly for the systemic disease and its lesions, and stated that search of the literature showed no unequivocal record of sarcoidosis coexistent with malignant neoplasia.

It appears to us that there is such an association in our two cases, which differ in the pattern of their medical histories and in their pathological features from previously reported cases of carcinoma with accompanying localized sarcoid-like granulomata. Both points establish the existence of long-standing systematized sarcoidosis, apparently independent of the bronchial new growth. Whether this association between the two conditions is fortuitous or whether it betrays an aetiological link cannot be said on the evidence available. The notion of a $\overrightarrow{\overrightarrow{0}}$ causal affinity between neoplasia and pre-existing disease of another sort, especially chronic irritation or chronic inflammation, is an old one and it is a possibility here which cannot be excluded a priori. Certain pneumoconioses (Weller, 1929; Merewether, 1949; Ellman, 1953) and tuberculosis $\tilde{x}$ (Wolf, 1895; Ewing, 1935) have often been held $\frac{5}{3}$ suspect in relation to bronchial carcinoma, and the recent studies of Raeburn and Spencer (1953), who claimed that malignant changes can be seen to develop first in post-inflammatory scars in the lung, 을 seem to offer support to the general hypothesis.

\section{SUMmaRY}

The histories of two patients with sarcoidosis of some years' duration and more recent pulmonary neoplasm are presented.

\section{REFERENCES}

Anderson, W. M. (1942-3). J. Ihorac. Surg., 12, 351.

Cowdell, R. H. (1954). Quart. J. Med., 23, 29.

Ellman, P. (1953). Proc. roy. Soc. Med., 46, 851

Eliman, P. (1953). Proc. roy. Soc. Med., 46, 851.

Ewing, J. (1935). Bull. N.Y. Acad. Med., 11, 281.

Fried, B. M. (1935). Amer. J. Surg., 29, 261.

Gherardi, G. J. (1950). Arch. Path., Chicago, 49, 163.

Merewether, E. R. A. (1949). Quoted by Ellman, vide supra

Nadel, E. M., and Ackerman, L. V.(1950). Amer. J. clin. Path.,20, 952

Nickerson. D. A. (1937). Arch. Path., Chicago, 24, 19.

Prior, J. T. (1952). Amer. J. Surg., 83, 201.

Raeburn, G., and Spencer, H. (1953). Thorax, 8, 1.

Symmers, W. St. C. (1951). Amer. J. Path., 27, 493.

Wolf, K. (1895). Fortschr. Med., 13, 725 and 765. 